\title{
Changing Scenario of Dentistry with COVID-19-An Overview
}

\author{
Ashima Trivedi ${ }^{1}$ and Ashwani Kumar Trivedi ${ }^{2}$ \\ ${ }^{1}$ Resident, Trivedi Dental Centre, Jabalpur, MP, India \\ ${ }^{2}$ Senior Resident, Trivedi Dental Centre, Jabalpur, MP, India \\ *Corresponding Author: Ashima Trivedi, Resident, Trivedi Dental Centre, \\ Jabalpur, MP, India.
}

Received: May 29, 2020

Published: June 29, 2020

(C) All rights are reserved by Ashima Trivedi and Ashwani Kumar Trivedi .

\begin{abstract}
Corona virus disease 2019 which proved to be a global pandemic has a noticeable impact on the field of dentistry. This is an overview of the changing scenario of dentistry with Covid-19 and the possible protocols which the Dental health care professional (DHCP) needs to follow for protection and safety in future. It covers all the changes and guidelines for dentists to continue with the dental practise. Also, the impact of novel corona virus has lead to a fear in public and professionals which may be noticed as a markable decline in dental treatments.
\end{abstract}

Keywords: Dentistry; Covid-19; Changing Scenario

\section{Introduction}

Coronavirus disease 2019 (COVID-19) may be defined as illness which is caused by a novel coronavirus, now referred to as severe acute respiratory syndrome coronavirus 2 (SARS-CoV-2; formerly called 2019-nCoV). Wuhan city, Hubei Province, China first identified amid an outbreak of respiratory illness cases. It was on January 30, 2020 where in WHO announced COVID-19 as a global health emergency. WHO declared COVID-19 as a global pandemic on March 11, 2020, which was the first such designation till now since the former body declared H1N1 influenza as a pandemic in 2009.

\section{Route of transmission}

Transmission occurs through respiratory droplets from coughing and sneezing, also some other respiratory pathogens are associated with it such as influenza and rhinovirus. Virus released in respiratory secretions are to known infect different individuals through direct contact with the oral cavity and mucous membranes.

\section{Presymptomatic/asymptomatic Covid-19 infection}

Data has also suggested that asymptomatic patients also have the ability to transmit infection. Therefore, this raises primary concerns for the effectiveness and need for isolation.
Studies have found that viral expression is through infection through nasal and throat swabs. Also increases in viral loads at the time when patients became symptomatic.

\section{Clinical manifestations}

Manifestations of COVID-19 have ranged from asymptomatic or mild symptoms to severe illness and lastly to mortality.

Symptoms have a tendency to develop from 2 days to 2 weeks following exposure to the virus.

Cough and shortness of breath or difficulty breathing in association with at least two of the following symptoms may positively indicate COVID-19:

- Fever

- Chills

- $\quad$ Repeated shaking with chills

- Headache

- Muscle pain

- Sore throat

- New loss of taste or smell. 
Other reported symptoms have included the following:

- $\quad$ Fatigue

- $\quad$ Sputum production

- $\quad$ Malaise

- Diarrhea

- $\quad$ Respiratory distress.

\section{Risks associated with dentistry}

The practice of dentistry involves the use of rotary dental and surgical instruments, such as handpieces or ultrasonic scalers and air-water syringes. These instruments can create a visible spray which comprises of particle droplets of saliva, water, blood, microorganisms, and other debris. Surgical masks can protect mucous membranes of the mouth and nose from droplet spatter, but they do not provide complete protection against air borne infections and inhalation.

Influence of COVID-19 on dentistry

Management of the patient

- $\quad$ Pre-screening of every patient with any symptoms associated with COVID-19.

- $\quad$ Ensuring social distancing in dental clinics and also efforts should be made to limit the people in the waiting area.

- Mandatorily use of a face mask for very patient and accompanying person.

- $\quad$ Systemic assessment of every patient upon arrival, which necessarily includes body temperature.

\section{Equipment considerations}

- Dental unit waterlines are to be tested for quality according to Environmental Protection Agency $(<500 \mathrm{CFU} / \mathrm{mL}$ ) to ensure safe drinking water.

- For autoclaves and instrument cleaning devices, routine cleaning and maintenance has to be carried out as recommended by the manufacturer.

- Follow protocol for storage and recommended maintenance as per manufacturer for devices such as air compressor, suction lines and vacuums, radiography equipment, amalgam separators, high-tech equipment and other dental equipment.
Administrative controls and work practices

- $\quad$ Efforts should be made to clinical and treatment care to a single patient at a time whenever possible.

- Clinics should be set up in such a manner that only the sterile supplies and instruments which are needed for the dental procedure are accessible, and all other non-essential supplies and instruments are covered and kept in storage, such as cabinets and drawers, and necessarily away from potential contamination.

- Aerosol-generating procedures are avoided as much as possible. Limit the use of dental handpieces and the air/ water syringes and prioritize minimally invasive dentistry with hand instruments only.

- If aerosol-generating procedures are necessary for dental care, use of four-handed dentistry is recommended with the use of a high evacuation suction and dental dams to minimize droplet spatter and aerosols

- Use of preprocedural mouth rinse by the patient with an antimicrobial product such as chlorhexidine gluconate, essential oils, povidone-iodine or cetyl pyridinium chloride to reduce the microbial load in the oral cavity.

\section{Engineering controls}

- Maintenance of all the ventilation systems that provide air movement from a clean area to contaminated flow direction is to be maintained properly.

- Use of a portable High Efficiency Particulate air filtration unit is recommended when the patient is undergoing dental treatment, with an aerosol-generating procedure.

\section{Patient placement}

- Dental treatment should be provided in individual rooms for every patient.

- If the treatment area has multiple chairs in same room, at least 6 feet of space between patient chairs.

- Also, physical barriers can be placed in between the dental chairs.

\section{Patient volume}

The maximum number of patients are determined who can undergo dental treatment at the same time in the dental facility, based on the layout of the facility number of room, and the time required to disinfect patient operatories after each patient. 
Strict adherence to hand hygiene practice

- Before and after every patient contact

- $\quad$ Contact with potentially infectious material

- Before putting on and after removing personal protective equipment (PPE), including gloves.

Using personal protective equipment (PPE)

There are multiple sequences recommended for donning and doffing PPE. One suggested sequence for DHCP includes:

Before entering the treatment area:

1. Perform Hand hygiene.

2. Put on a protective clothing which covers personal clothing and skin.

3. Put on a surgical mask or respirator.

4. Put on eye protection.

5. Perform hand hygiene.

6. Put on clean non-sterile gloves.

7. Can put double gloves if needed.

8. Enter the treatment area.

After completion of the dental treatment:

1. Remove gloves.

2. Remove protective clothing and discard the gown in a container.

3. Exit the patient room or care area.

4. Perform hand hygiene.

5. Remove eye protection.

6. Remove and discard surgical mask or respirator.

7. Perform hand hygiene.

Education and training

- Dental Health Care Professional should be provided with a job or task-specific education and training on preventing transmission of infectious agents.

- Ensuring that Dental Health Care Professional are well educated, trained, and have practiced the correct use of PPE prior to the treatment, and prevention of contamination of clothing, skin, and the environment during the process of removing such equipment.

\section{Conclusion}

Episode of Covid-19 has a remarkable impact on dentistry. Guidelines issued are to be followed by dental health care professional for personal and community's safety. There can also be a fear among dental fraternity amid the Covid-19 which may lead to decline in elective dental treatments. Also, the maintenance of dental operatory tends to increase in cost and proves to be a burden on dental health care professional.

\section{Acknowledgement}

Dr. Rajshri Trivedi, Dr. Anant Trivedi

\section{Assets from publication with us}

- Prompt Acknowledgement after receiving the article

- Thorough Double blinded peer review

- Rapid Publication

- Issue of Publication Certificate

- High visibility of your Published work

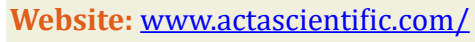

Submit Article: www.actascientific.com/submission.php

Email us: editor@actascientific.com

Contact us: +919182824667 